RAJEEV BATRA, Y. CHARLES ZHANG, NILÜFER Z. AYDINOĞLU, and FRED M. FEINBERG*

Building on cultural values research, the authors identify specific image attributes on which multicountry brands should position themselves consistently across markets. Leveraging prior research, they identify three life values that are most equal (benevolence, universalism, and self-direction) and two that are least equal (power and hedonism) in cross-national importance. The authors link specific brand image attributes (e.g., friendly, social, elite style, arrogant) to these life values through empirical data and semantic analysis. Using an extensive field data set on consumer perceptions and preferences from 22 countries regarding more than 1,700 brands, the authors then show that greater global consistency of a brand's image decreases overall brand attitudes if the specific image attribute is one that is not equally desired worldwide. They also find that the attitudinal impact of a multicountry brand's positioning consistency on commonly valued image attributes is greater when the set of competitors the brand faces across its markets is more homogeneous. The authors discuss implications for global brand management theory and practice.

Keywords: cross-cultural values, global brands, international marketing strategy, brand image

\title{
Positioning Multicountry Brands: The Impact of Variation in Cultural Values and Competitive Set
}

"Some brands have established a strong, consistent connection across cultures by tapping into fundamental human truths: the commonalities that unite rather than

*Rajeev Batra is S.S. Kresge Professor of Marketing, University of Michigan (email: rajeevba@umich.edu). Y. Charles Zhang is Assistant Professor of Marketing, Anderson Graduate School of Management, University of California, Riverside (email: charles.zhang@ucr.edu). Nilufer Z. Aydinoğlu is Associate Professor of Marketing, Koç University (email: naydinoglu@ku.edu.tr). Fred M. Feinberg is Joseph Handleman Professor of Marketing and Professor of Statistics, University of Michigan (email: feinf@umich.edu). The authors gratefully acknowledge the research assistance of Tanya Zhang. Mr. Ed Lebar of Young \& Rubicam Brands Knowledge Group was very kind in allowing use of these BAV data, and the crucial help and comments of David Dyte, Emina Hrustic, and Steven Root from Y\&R are also much appreciated. The authors also acknowledge the comments and other help by colloquium attendees at the Nanyang Busines School, Singapore, and the University of California, Irvine, as well as Dana Alden, Richard Gonzalez, Aradhna Krishna, Puneet Manchanda, Daphna Oyserman, Jan-Benedict Steenkamp, and Stefan Wuyts. Coeditor: Robert Meyer; Associate Editor: Sanjay Sood. divide people across the globe, such as the desire for love... and happiness. Such a platform opens up real opportunities to make your marketing budget work more efficiently, but finding the right idea can be a tough challenge."

-Hollis (2008, p. 165)

A key challenge facing multinational marketers today is to devise the best positioning strategy for global brands across multiple national markets, which often vary in their cultural values as well as in their competitive contexts (Torelli et al. 2012). These multicountry brands are typically marketed across multiple countries in a very similar and coordinated way, utilizing consistent associations and image positioning (Steenkamp, Batra, and Alden 2003, p. 53). Such cross-national marketing standardization can lower total global costs (through economies of scale; Samiee and Roth 1992), speed up market rollout (Neff 1999), and improve consumer preference by creating a positive perception of "globalness" (Steenkamp, Batra, and Alden 2003). However, it can also decrease local market relevance (Craig and Douglas 2000). 
The literature on the standardization versus adaptation of international marketing strategy has long argued that only a high level of similarity in consumer needs and competitive contexts should justify the kind of standardization that can lower costs without hurting consumer preference (e.g., Zou and Cavusgil 2002). However, the extent of homogeneity of markets on consumer needs and wants and the degree of standardization by brands on marketing strategy dimensions have not received adequate attention in the literature, despite their centrality to the focal research issue (Ryans, Griffith, and White 2003, pp. 593-97). Nor has prior literature attempted to identify the specific consumer needs that are similar enough across countries to justify standardizing on them. It has also not empirically tested the impact on standardization of competitive similarity, using market data. In this article, we complete the aforementioned tasks, taking advantage of the unique and large multicountry Brand Asset Valuator (BAV) database and building on previous research on cross-country variations in cultural values.

Despite the recent growth of "global consumer culture" (Alden, Steenkamp, and Batra 1999), studies still find very significant differences across countries in their cultures and values (e.g., Hofstede 2001; Schwartz 2004), suggesting countervailing local or hybrid cultural affinities (Hannerz 1990). A review chapter by Gupta, Winkel, and Peracchio (2009) provides a vivid example of how the standardized positioning of global brands can encounter problems as firms cross cultural boundaries. Apple's ad campaign showing opposing Mac and PC personalities became widely liked in the United States for depicting Macs as more fun and play-oriented and PCs as more efficiency-focused, rule-following, and work-oriented. However, this brand positioning was received much more negatively by consumers in Japan. Because of different cultural values, "Japanese consumers indicated that the PC's sacrifice for group conformity, work ethic, and pride in the organization were positive values, much more positive than the fun and approachable benefits offered by the Mac" (Gupta, Winkel, and Peracchio, p. 230).

Such differences in cultural values are a critical obstacle that global marketers must overcome in their attempts to develop economically viable, yet locally relevant, multicountry brands. Several researchers have noted that many brands offer not just functional benefits but also linkages to deeper cultural values through their abstract qualities (e.g., Shavitt, Torelli, and Wong 2009; Torelli and Kaikati 2009). Global brands, in particular, frequently are carriers and symbols of culture (Torelli et al. 2012).

Several prior cross-cultural studies have shown that brand marketing strategies using culture-congruent appeals tend to outperform those lacking such congruence (Yavas, Verhage, and Green 1992; Zarantonello, Jedidi, and Schmitt 2013). For instance, Aaker and Lee (2001, Experiment 2) showed that ads using a more "promotion-focused" message (highlighting possibilities for advancement) do better in cultures where consumers are higher in independent self-construal (e.g., North America), whereas ads with a more "prevention-focused" message (highlighting security and safety) do better where consumers are higher in interdependent self-construal (e.g., East Asia). Thus, global brands that are highly symbolic of particular cultural values could easily evoke positive attitudes in matching cultures, but not in mismatched cultures (as in the previous Apple example). Given the similarities and differences in cultural values across their many national markets, the ideal solution for global brands would be one in which they position themselves consistently across markets on just those brand meanings that are themselves highly valued in (almost) all markets and cultures.

In this article, we therefore contribute to the literature streams on cross-cultural values and global brand strategy in multiple ways. First, through a deep and novel integration of multiple literatures on cross-cultural values, we identify which specific values are considered uniformly more important across more cultures, and for what functional and evolutionary reasons. Second, we apply these conclusions to the substantive domain of global brand management, proposing and showing the differential impact on attitudes toward a global brand if it is positioned consistently on consumer needs and values that are more similar (vs. varied) in their importance across multiple countries. Here, we analyze data on the extent of homogeneity across markets on specific values, and the extent to which specific brands are positioned consistently on these, which has never been done before. We also explore for the first time the moderating influence of competitive set heterogeneity on the effectiveness of standardized global positioning strategy. Drawing on our findings, we show how global brands can do a better job of balancing the economic and speed advantages of standardization with the need for local market relevance by strategically positioning themselves consistently on only those specific, nowidentified attributes that consumers across the world desire more (vs. less) uniformly.

From a competitive positioning perspective, a multicountry brand that faces a more varied set of competitors across its multiple markets is also likely to face a more varied set of competitive brand positioning platforms to which it needs to respond. The international marketing strategy literature has therefore also discussed the importance of the similarity of the competitive environment in driving standardization decisions (Jain 1989; Samiee and Roth 1992; Zou and Cavusgil 2002), proposing that standardization should be more appropriate if the firm competes with the same rivals in different markets. However, empirical examination of this proposition has not yet appeared in the literature.

In this article, we therefore test the attitudinal impact of the nature and consistency of the specific consumer values used in a multicountry brand's positioning, as well as that of the competitive context it faces across markets. To do so, we take advantage of the BAV database, arguably the most comprehensive global database of consumer perceptions on brands, containing disaggregate perceptual and attitudinal data on multicountry brands as well as data on their competitors worldwide. Our analysis sample consists of field data from 64,790 consumers on 1,723 brands competing cross-nationally in 22 countries. Though not designed to test our research questions, data of this scope provide a rare and valuable opportunity to conduct this important academic investigation. We supplement these BAV data by additional cross-national studies conducted at the consumer level that enable us to match the BAV brand imagery data to the types of consumer values they best represent.

In the following section, we develop the theoretical foundations of our research. We first discuss how cross-cultural brand positioning uses image attributes designed to symbolize cultural values. Next, we present both theoretical arguments and empirical results from the cross-cultural values literature showing which specific values should and should not vary in their cross-cultural importance, in line with their adaptive social functions. We then describe in detail our proprietary BAV data 
set and the way in which we link specific brand image attributes to particular cultural values using prior research and our own multicountry consumer studies. After our analyses and results, we conclude by discussing implications and our contributions.

\section{LITERATURE REVIEW AND \\ CONCEPTUAL DEVELOPMENT}

Cross-Cultural Brand Positioning: The Influence of Values

Before we examine how cross-cultural differences in consumer values can hurt global brands, it is necessary to discuss how brands are often chosen for their symbolic and image attributes (Aaker 1997). By "image attributes," we mean perceptions of symbolic aspects of the brand that include its brand personality (i.e., its human-like perceptions of being friendly, honest, upper-class, exciting, etc.; Aaker 1997), perceptions about the types of people who use it (Dichter 1985), and intangible associations such as innovativeness and reliability. Such abstract qualities are especially important in cross-cultural research because of global brands' ability to communicate cultural meaning across markets (Aaker, Benet-Martínez, and Garolera 2001; Torelli et al. 2012).

We follow prior literature (e.g., Torelli et al. 2012) in conceptualizing these brand image attributes as being symbolic of deeper cultural and societal values. Values are defined as "desirable, trans-situational goals, varying in importance, that serve as guiding principles in people's lives" (Schwartz and Bardi 2001, p. 4). They are conceptions of desirable end states that reflect what people regard as important in life, including preferences across brands and brand attributes (Torelli and Kaikati 2009). Batra, Homer, and Kahle (2001) find that values influence brand attitudes by prioritizing the importance of product and image attributes to consumers. For example, consumers higher on "other-directed values" (e.g., being wellrespected, having a sense of belonging) place a greater importance weight on brand image attributes such as reputation and style, whereas consumers higher on "self-directed values" (e.g., self-fulfillment, self-respect) place a greater importance weight on attributes such as care and product fit (Batra, Homer, and Kahle 2001, p. 123). Other researchers (e.g., Aaker, BenetMartínez, and Garolera 2001, pp. 494-95) have identified conceptual linkages between specific brand image attributes (e.g., sincere, exciting, sophisticated) with specific types of life values (e.g., security, excitement, status/prestige). Thus, variations across countries in the importance placed on particular life values should lead to differences in the brand image attributes these countries consider important. We next turn to research on how societies differ in the importance they place on these underlying values.

\section{Cross-National Commonalities in the Importance of Specific Values}

Substantial prior research has documented variations in the desirability (i.e., importance ratings) of life values across people from different cultures (e.g., Hofstede 2001; Schwartz 1992, 2004). Value dimensions studied include individualism versus collectivism, power distance, horizontal/equality versus vertical/ hierarchy orientations, masculinity/mastery versus femininity/ nurturance, uncertainty avoidance, and independent versus interdependent self-construals (for reviews, see Gupta, Winkel, and Peracchio [2009] and Shavitt et al. [2006]). Perhaps the broadest examination of these cross-national value patterns is in work by Schwartz (1992, 2004) and Schwartz and Bardi (2001). They develop a cross-cultural values typology using data from 1988 to 1996 from over 60 nations. Data were collected on the subjects' rated importance of 57 individual values (e.g., social status and prestige, safety, harmony and stability of society), and 45 of these were subsequently collapsed into ten multi-item "value types" (Schwartz 2004).

The ten Schwartz value types (with sample items in parentheses), are POWER (status, prestige, authority), ACHIEVEMENT (successful, ambitious, influential), HEDONISM (pleasure, enjoying life, sensuous gratification), STIMULATION (excitement, daring, novelty), SELF-DIRECTION (independent thought, creativity, freedom), UNIVERSALISM (equality, unity with nature, broadminded), BENEVOLENCE (helpful, honest, forgiving), TRADITION (respect for tradition, devout, accepting my position in life), CONFORMITY (obedience, self-discipline, honoring parents and elders), and SECURITY (safety, social order). The value types found in Schwartz's research have been shown to map well onto the dimensions studied by other researchers (Aaker, Benet-Martínez, and Garolera 2001; Inglehart and Oyserman 2004). However, to our knowledge, they have never been used in research on the positioning strategies of multicountry brands.

Theoretical arguments for cross-national ranking of importance means. Schwartz $(1992,2004)$ and Schwartz and Bardi (2001) find a striking degree of consensus across people in different societies in the relative importance given to these ten value types. As pointed out by Schwartz and Bardi, there are strong reasons to expect this, given the "shared bases of values in human nature and the adaptive functions of each value type in maintaining societies" (p. 280). A basic social function of values is to motivate and control group members to best promote group survival and prosperity (Parsons 1951). Thus, the relative importance societies place on these ten value types should be driven by their differential utility in supporting group welfare and interests.

Schwartz and Bardi (2001, pp. 280-82) theoretically propose that societies use values to help meet three key requirements, prioritized in this sequence of importance: (1) ensuring group survival, by promoting cooperative and supportive relations among members of primary groups; (2) seeking societal prosperity, by motivating people to invest time and effort to perform productive work, solve problems, and generate new ideas; and (3) allowing for (and legitimizing) the gratification of selforiented needs and desires, so that members do not get frustrated and withdraw from investing in group goals. The theoretical reason for this prioritization - and thus, for the relative importance of these ten value types-is that societal survival and security needs are naturally of primary concern, coming before secondary and tertiary concerns about individual quality of life and self-expression (Inglehart and Oyserman 2004). A growing body of recent research (e.g., Gelfand et al. 2011; Henrich 2004; Norenzayan and Shariff 2008; Norenzayan et al. 2016) has argued that less evolved societies that face more existential threats logically give primacy to survival needs and the social values that ensure survival before they begin to care about individual and self-expressive needs.

Given this societal role of values and this prioritization of societal needs, it follows logically that the values of BENEVOLENCE (toward one's primary group members) are most critical for group cooperation and survival, followed by UNIVERSALISM (with its broader focus on all others). SELF-DIRECTION values 
can also be expected to be highly ranked across cultures because they motivate people to work productively, foster innovative solutions to problems, contribute to prosperity in normal times, and are especially "crucial to meeting the challenges posed by change in times of crisis" (Schwartz and Bardi 2001, p. 282). Schwartz and Bardi (2001) argue that because such self-direction values provide an intrinsic source of motivation, their pursuit need not come at the expense of others in society; this value type thus advances the second and third group priorities without undermining the first. Thus, in the Schwartz framework we adopt here, SELF-DIRECTION values are very important in less evolved societies as well, because rejecting them would create individual frustration and withdrawal of investment in group tasks (Schwartz and Bardi 2001, pp. 280-83).

SECURITY and CONFORMITY, which help avoid risk and control forbidden impulses, serve to maintain the status quo and thus come next in line in Schwartz and Bardi's (2001) explanation of the relative societal prioritization of these values. These two value types are relatively less important than the first group because they do interfere with people's ability to find innovative solutions to problems and gratify self-oriented needs and desires. ACHIEVEMENT values are also placed here (but not higher) in relative priority, because they can motivate people to invest time and energy in tasks that serve group interests but also have the potential to motivate selfenhancing behaviors that can disrupt harmonious positive social relations.

POWER, HEDONISM, and STIMULATION values, in contrast, should have the least relative societal importance for the simple reason of "their irrelevance for the first two group requirements" (i.e., societal priorities; Schwartz and Bardi 2001, p. 282). Although these three value types are relevant to the third-listed societal priority of helping meet self-oriented needs (of physical gratification and optimal arousal), they are the most likely to threaten positive social relations (POWER most of all, as it implies dominance over people and resources, which potentially entails harming or exploiting others). ${ }^{1}$ TRADITION should also belong to this lowest-importance group, because although it contributes to smooth group functioning, it pertains to abstract beliefs more than it does to daily social behavior, reducing its societal relevance.

Empirical support for cross-national ranking of importance means. Providing empirical support for this theoretically developed importance prioritization, Schwartz and Bardi (2001) show in their extensive cross-national data that values of BENEVOLENCE, SELF-DIRECTION, and UNIVERSALISM are observed to be (almost everywhere) much more highly ranked (1, 2, and 3, respectively) than values of HEDONISM, STIMULATION, TRADITION, and POWER (ranked 7, 8, 9, and 10 , respectively), with values of SECURITY, CONFORMITY, and ACHIEVEMENT being ranked in the middle $(4,5$, and 6 , respectively). (For these importance means and ranks, taken from Schwartz and Bardi [2001, Table 3, p. 275], see the Appendix.)

1Torelli and Shavitt (2010, p. 705) have argued that the meaning and purpose of POWER values can differ across cultures, in some cases serving nurturant goals. Here, we limit ourselves to the conceptualization and measurement of POWER as "social status and prestige, dominance over people and resources (social power, authority, wealth, preserving public image)" (Schwartz and Bardi 2001, p. 270). Torelli and Shavitt (2010, p. 704) note that Schwartz's perspective has a strong relationship with authoritarianism and "is a good fit to a self-centered power concept."
Variations in the Importance of Specific Values Across Cultures

The previous discussion speaks to the universal logic for the mean rankings that emerge when the Schwartz and Bardi (2001) data are examined across nations. At the same time, we also observe that in these data, although the consistency in importance means is high for some value types, there is less consistency for other types. The published data of Schwartz and Bardi (see Appendix) also provide these variations (standard deviations [SDs]) of the importance ratings of value types across countries, not just the means. We first present this empirical evidence, followed by a theoretical explanation.

Empirical evidence on variation in values' importance. Schwartz and Bardi's (2001) largest sample comes from teachers/ students from 56/54 countries. In these data, the degree of crosscountry consistency (SDs) of rated importance of value types varies from roughly .25 at the low end to approximately .65 at the high end, a range of .40 (on seven-point scales). Creating four quartiles within this SD range enables us to put these ten value types into four groups. Thus, national cultures seem to vary the least in their rated desirability (i.e., have the lowest SDs) for the life values of BENEVOLENCE (.28/.25), UNIVERSALISM (.31/.29), SELF-DIRECTION (.31/.31), and ACHIEVEMENT $(.31 / .30)$ and vary the most (i.e., have the highest SDs) for POWER (.55/.43) and HEDONISM (.59/.65), with the rest falling in the two middle quartiles. ${ }^{2}$

Theoretical explanation for cross-national variation in values' importance. Why might some value types be rated higher in some countries than others, while other value types stay relatively stable? Although there could be multiple explanations, the literature has suggested that the natural evolution of societies ought to lead to changes in the importance placed on different values, in line with the adaptive functions served by these values.

A growing body of multidisciplinary recent research has argued that the values and norms that societies develop are an evolutionary adaptation to the threats they face and the resources they possess, with societies that develop the most ecologically useful values and norms out-competing others in this process of natural selection and advancement. Thus, Henrich (2004) argues that prosociality values develop in order to restrain uncontrolled aggressive behavior, while Gelfand et al. (2011) show empirically that, among 33 societies, those that face greater threats (e.g., population pressure, resource scarcity, conflict, natural disasters) tend to be "tighter" cultures, where social norms and sanctions (i.e., "felt accountability") are stronger. According to Norenzayan and Shariff (2008) and Norenzayan et al. (2016), when a society's deities embody moral values and are believed to reward/punish human behavior, its members are less likely to freeload and more likely to show social solidarity and cooperation.

Thus, less evolved societies facing more existential threats logically give primacy to survival needs and the social values that ensure survival. Societies where these first-order priorities are met then begin to focus more on their secondary and tertiary

2 We organize these data by quartiles because tests of the moderating effect of a continuous variable are frequently performed by creating four quartiles and testing for differences across the first and last quartiles, or by creating thirds and testing for differences across the top and bottom thirds (e.g. Chandon and Wansink 2007). Issues with this "extreme groups approach" are discussed by Preacher et al. (2005). 
societal needs (Inglehart and Oyserman 2004; Schwartz and Bardi 2001). According to a time-series analysis discussed by Inglehart and Oyserman (2004), as economies develop and prosper, societies move from a focus on collective economic and physical security, hard work, and shared societal goals toward an emphasis on personal autonomy, self-fulfillment, and the pursuit of individual pleasure and an exciting life. Accordingly, as social structures evolve and mature, and after group survival is ensured and societal prosperity is secured, the gratification of self-oriented needs and desires gains importance. Values serving secondary and tertiary functions thus gain in importance in a sequential fashion, while the importance of values that serve primary functions remains high (Schwartz and Bardi 2001). Thus, it follows logically that societies at different levels of development should evidence more consensus on the importance of values serving first-order functions (e.g., BENEVOLENCE) and be less consistent in the importance they place on cultural values serving lowerorder functions (e.g., POWER). ${ }^{3}$

These data from Schwartz and Bardi (2001), and the aforementioned theoretical frameworks that account for them, thus offer very useful insight into the specific nature of values that vary in the degree to which they "unite" us (i.e., are more uniformly desired) versus "divide" us (i.e., are less uniformly desired). As Ryans, Griffith, and White (2003) point out, this is a crucial variable that prior research in the standardization (global marketing strategy) literature has not yet empirically utilized. We contribute to this literature by drawing and testing the implications for global brand management of these consequent variations in the cross-national consistency of societal values.

Combined, the theoretical explanations and data suggest the following:

$\mathrm{H}_{1}$ : Multicountry brands gain in overall cross-national attitudes by being consistently positioned on image attributes (and underlying values) that are themselves desired most equally across markets. In contrast, multicountry brands lose in overall attitudes by being consistently positioned on image attributes that vary the most in desirability across markets.

This should happen because, as discussed previously, attitudinal responses to the brand should be positive everywhere for universally important values, leading to high overall brand attitudes. For values that vary in their cross-national importance, however, attitudinal responses should be high in some countries but low in others, leading to relatively lower overall cross-national brand attitudes.

For theory-testing purposes, we limit ourselves to the values at the extreme first and fourth quartiles of the SD distribution:

$\mathrm{H}_{1 \mathrm{a}}$ : High cross-national consistency of a brand on image attributes reflecting consumer values of BENEVOLENCE,

${ }^{3}$ We note that most of the specific values that have low importance SDs also have high importance means (e.g., BENEVOLENCE), and that most of the values that have high importance SDs also have low average importance mean scores (e.g., POWER). This is theoretically reasonable: because values such as POWER are relatively lower in the importance prioritization in less evolved societies (Schwartz and Bardi 2001), and gain in importance only with societal evolution, becoming higher-rated only in the smaller subset of more evolved societies, they should naturally end up with both lower average importance means and higher cross-national SDs. In our "Analysis" section, we address the methodological implication of this near-confounding of SDs with means.
UNIVERSALISM, SELF-DIRECTION, and ACHIEVEMENT

are positively associated with overall brand attitudes. ${ }^{4}$

$\mathrm{H}_{1 \mathrm{~b}}$ : High cross-national consistency of a brand on image attributes reflecting consumer values of POWER and HEDONISM are negatively associated with overall brand attitudes.

\section{Exploring the Moderating Influence of Competitive Context}

The literature on international marketing strategy has emphasized the important impact of the competitive environment's cross-market variation on standardization decisions, arguing that when a multimarket brand faces a more varied set of competitors, standardization of its own image-positioning strategy may be detrimental for performance. Specifically, Jain (1989, p. 74) cites theoretical arguments in the prior literature (Copeland and Griggs 1985; Quelch and Hoff 1986) to propose that "if a firm competes with varying rivals in different markets, it should lead to reduced standardization in the focal firm's marketing strategy." Samiee and Roth (1992) list the presence of similar standardized products marketed by the firm's major competitors as one of the external factors that favors a more globalized marketing strategy. This is also in line with the theoretical arguments in the economics and competitive strategy literature streams on "multipoint competition" (where firms compete in more than one market simultaneously; Gimeno 1999; Karnani and Wernerfelt 1985).

The set of competitors a multicountry brand faces often varies: the German chemical and consumer goods company Henkel, for instance, has found that its set of top three competitors is different in each of its six major European markets (Arnold and Schroiff 2004). As an example from our BAV data, we find that the consumer electronics brand Leica faces mostly similar competitors in Germany and Japan (e.g., Pentax, Samsung, Sharp, Sony, Minolta), with only a few that differ (Grundig, Practica, Schneider, and Nordmende are competitors only in Germany). The number of varying competitors is much greater in our data for the financial services brand BNP. Although it faces some of the same competitors in France and Japan (e.g., American Express, Citibank, Mastercard/Eurocard, Visa), it faces many more locally varying but still strong competitors in these markets (e.g., Japan: Shinsei Bank, Saison, Nippon Shimpan; France: Bank Populaire, Carte Bleue, Credit Lyonnaise).

It is a fundamental marketing strategy principle that a brand that does not respond to competitive positioning platforms likely loses out in terms of consumer attitudes (e.g., Carpenter, Glazer, and Nakamoto 1994; Van der Lans, Van Everdingen, and Valentyna 2016). Therefore, a multicountry brand that faces greater competitive heterogeneity - and thus, a greater potential range of competitive positioning platforms - should logically vary its own brand positioning more across these markets. As an example, Samsung mobile phones face lower-priced Chinese competitors such as Huawei and ZTE in Nigeria but face premium competitors such as the Apple iPhone in the United States. Using an "affordable" image positioning in Nigeria but a "premium" one in the United States - thus sacrificing global consistency — might increase overall global attitudes more for Samsung than a strategy that insists on a consistently premium position worldwide.

${ }^{4}$ We include ACHIEVEMENT in $\mathrm{H}_{1 \mathrm{a}}$ because it, along with the other three values listed with it, falls within the lowest quartile of SDs in the Schwartz and Bardi (2001) data that we use here (see footnote 3). Our data (discussed subsequently), did not, however, allow us to test this hypothesized effect for ACHIEVEMENT. 
Despite this prior recognition of the importance of competitive consistency as a determinant of the standardization decision for multimarket brands (e.g., Jain 1989), it has not been empirically tested with objective market/consumer data. Nor has it been tested empirically with market data in the "multipoint competition" literature (beyond some simulation results). Given that the BAV data set provides us the opportunity to empirically calibrate the degree of competitive set heterogeneity across markets, we thus explore in these data how the relationship between the focal brand's degree of overall image consistency and its overall consumer attitudes varies with the heterogeneity of its competitive set across its markets. We also explore how this moderating effect of competitive set heterogeneity might further vary with the nature of the cross-cultural values (image attributes) on which the focal brand is consistently positioned. (Because the details of these analyses cannot be accommodated within this article, we provide a summary here and detail them in the Web Appendix.)

\section{DATA SETS AND PRELIMINARY ANALYSES}

The main data that we use come from the Young \& Rubicam (Y\&R's) proprietary BAV database. These data are collected for commercial use to track how consumers in multiple countries perceive brands of interest, and they are not designed to test hypotheses such as ours. Although this creates several difficulties (described subsequently), these BAV data nonetheless provide a rare and valuable opportunity to test our theory-based hypotheses, with important potential implications for global brand management theory and practice. Our portion of this field data consists of perceptual (image) and attitudinal ratings from 64,790 consumers in 22 representative countries on 1,723 brands competing cross-nationally in more than 27 broadly defined product and service categories. Next, we outline the details and characteristics of the data set and describe the data-preparation techniques. Then, we describe the additional research and data we used to link these BAV brand attributes to the Schwartz value types used in our theoretical development.

\section{BAV Data}

Countries, categories, and brands. We used BAV data from 22 countries in our analyses ${ }^{5}$ to include countries at different levels of economic development, from different regions of the world, with different social contexts; these data sets contained complete, comparable information on our variables. Several are large and growing "emerging markets" (e.g., Brazil, Mexico, India, China, Malaysia). We analyzed 1,723 brands that were present in at least two countries (thus qualifying them for the necessary "multicountry" status). Brand ratings were collected from respondents with no indication of product category. The BAV then classified the brands into (possibly several) microcategories, 224 in all. To allow our analysis to account for the category, we consolidated these into 27 macrocategories and

5 The countries from which we used BAV data included the United Kingdom $(3,614$ respondents), France $(2,327)$, Germany $(4,388)$, Holland $(1,501)$, Italy $(2,272)$, Poland $(2,503)$, Spain $(2,854)$, Sweden $(1,579)$, Australia $(3,841)$, New Zealand $(2,399)$, Brazil $(2,982)$, Chile $(2,481)$, Mexico $(2,980)$, Peru $(1,647)$, Uruguay $(1,879)$, India $(3,016)$, China $(5,033)$, Malaysia $(1,464)$, Thailand $(1,897)$, Japan $(2,219)$, Canada $(2,587)$, and the United States $(9,327)$. assigned every brand to the one category (e.g., personal hygiene) in which it had the highest usage across all countries.

Sample and data collection instrument. It is important to note that the same data were collected on each of these brands with standardized questionnaires, using similar data collection methods across the 22 countries. For example, in the United States, the BAV survey is administered quarterly (covering different brands); it uses 22 versions of a 24-page mail questionnaire (each covering a subset of the complete brand list), with response rates of $66 \%$ on average, resulting in approximately 6,600 respondents per quarter from a panel of about 10,000 respondents. In all countries, the samples are balanced by the local data collection vendor to match local census proportions on age, gender, and region. In non-English-speaking countries, standard back-translation procedures are employed to create the local-language questionnaire versions. Consumers rate multiple brands from multiple product categories. Although the goal is to cover all the major brands, not all brands in each category are rated; this is an unavoidable limitation of the BAV data. (As we explain further, we analyze the data at the level of brands, not individuals.)

Variables and constructs. Our data contain consumer ratings of these brands on various dimensions. In the BAV survey, brands that fulfill the initial minimum knowledge (familiarity) requisite (a nonzero level) are then rated on the variables we describe next, as well as a few other BAV variables (regard/ esteem and relevance). ${ }^{6}$ The data we use contain 48 brand attribute ratings, where consumers provide a "yes/no" response to a checklist of attributes, indicating whether (1) or not (0) they associate a certain characteristic with that particular brand. Some of these variables assess consumer perceptions of a brand's overall attitude-like perceptions such as "best brand," "worth more," "high performance," and "high quality." Others pertain to brand personality/image (Aaker 1997), in which brands are rated on selected personality adjectives such as "arrogant," "helpful," and "stylish." For a full list of constructs and variables in our data, see Table 1, with noted links to Aaker's (1997) work.

Data preparation. Our hypotheses are stated at the level of brands, which serve as our unit of analysis. Importantly, they are not stated at the level of individuals, who provided the binary (yes/no) responses. Furthermore, because these individual-level ratings are binary, they do not assess the degree to which consumers believe a brand possesses a given attribute. Therefore, to obtain brand-level and nonbinary measures suitable for analysis, we computed the proportion of respondents who associated a certain image attribute with a given brand separately for each country (e.g., 22\% of Canadians rated Brand X as "rugged"). Some of the 48 attributes were later combined into higher-order factors based on factor analysis, and some were used to measure brand attitudes; we describe these steps next.

Consistency of positioning for a given brand (our independent variable) could be assessed through the SD of the within-country proportions across countries (e.g., 22\% of Canadians, but only $10 \%$ of Japanese, rate brand X as rugged). A small SD would indicate that a brand is viewed similarly across

${ }^{6}$ These variables are used by BAV for its own purposes and were included in our analyses simply as control variables because we have no theory or hypotheses concerning them. 
Table 1

BAV VARIABLE DESCRIPTIONS

\begin{tabular}{|c|c|}
\hline Variable Name/Variable Group & Explanation \\
\hline Knowledge/Familiaritya & $\begin{array}{l}\text { Consumers' overall awareness of the brand; the extent of } \\
\text { understanding of what the brand stands for. }\end{array}$ \\
\hline Esteem/Regarda & $\begin{array}{l}\text { How highly consumers think and feel about the brand; the } \\
\text { extent to which consumers like a brand and hold it in high } \\
\text { regard. }\end{array}$ \\
\hline Relevance $^{\mathrm{a}}$ & $\begin{array}{l}\text { The breadth of a brand's appeal; the extent to which a brand is } \\
\text { perceived to be appropriate for a respondent's needs. }\end{array}$ \\
\hline Brand image attributes & 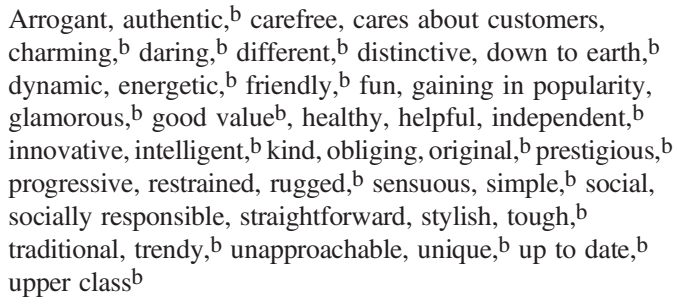 \\
\hline Brand attitudes & $\begin{array}{l}\text { Best brand, worth more, leader, high performance, reliable, } \\
\text { trustworthy, high quality }\end{array}$ \\
\hline
\end{tabular}

Measurement
$\begin{aligned} & \text { Seven-point scale question ("never heard of" to "extremely } \\ & \text { familiar") }\end{aligned}$

Seven-point scale question ("extremely low regard" to "extremely high regard")

Seven-point scale question ("not at all relevant" to "extremely relevant")

0 (no) or 1 (yes); checklist-type response style.

Items are treated individually or as multiple-item composites (see text and Table 2 for details).

\footnotetext{
aWe did not use these variables to test our hypotheses but instead used them only as control variables because models using BAV data typically include them.
Their cross-country consistency did not significantly affect brand attitudes in our models either as a main effect or in their interaction with brand image consistency. bItems appear in Aaker's (1997) list (with exact or very similar wording).
}

countries. Before calculating the cross-country SDs, however, we first needed to correct the within-country proportions for response style and scale-usage differences (i.e., differential rates of usage of the "yes" response category, by country; Baumgartner and Steenkamp 2001). Therefore, to make these within-country proportions amenable for cross-country analysis (Fischer 2004; Van de Vijver and Leung 1997), we performed the specific type of within-country standardization (ipsatization) recommended by Fischer (2004, p. 277; see Figure 1 and Web Appendix A). ${ }^{7}$

Factor-analytic data reduction and attitudinal dependent measure. As we have mentioned, the 48 original BAV items were subjected to exploratory and confirmatory factor analyses (EFAs and CFAs) to reduce possible collinearity and suggest logical multi-item scales for our subsequent regression analyses. These analyses yielded six CFA-validated factors, which we used in the subsequent regression analyses as multipleitem scales. (Possibly because of prior factor reduction by the BAV, 17 of the 48 items did not load on multi-item factors and were included in our analyses as single-item measures.) Details of these factor analyses appear in Web Appendix B.

In the first of the six CFA-validated factors, seven of the BAV items (best brand, high performance, high quality, reliable, trustworthy, worth more, and leader) were found to load together, capturing an overall "attitudinal positivity" assessment. Although this measure did not include more standard evaluative attitudinal items (e.g., good/bad), it clearly captures an overall evaluation of the brand (best brand, worth more, leader), which has been found to be a strong predictor of subsequent purchase behaviors. It also contains the kinds of summative assessments of underlying

${ }^{7} \mathrm{We}$ also estimated those models using interquartile ranges as an alternative measure of consistency, as well as SDs of arcsine-transformed proportions, with substantively similar results, which can be obtained from the authors. attributes (high performance, high quality, trustworthy, reliable) that attitude theory considers as contributing to such overall evaluations (for a review, see Wilkie [1994, pp. 280-307]). This brand attitudes scale $(\alpha=0.88)$ thus constitutes the dependent variable in our regression analyses, subsequently regressed on our image consistency data.

For brevity, we do not detail the five other multi-item brand image factors created using the CFA (labeled F for factor: F-Momentum, F-Elite style, F-Friendly, F-Rugged, F-Helpful), but they appear in Table 2. The table also reports, for each factor, the constituent items we used, as well as the reliabilities, the Fornell and Larcker (1981) average variance extracted measures of convergent validity, and discriminant validity test statistics; all met conventional criteria.

\section{Matching the BAV Data to Schwartz's Life Value Types}

Our conceptual development relies on Schwartz's life values. However, the BAV image attribute data we are using to operationalize them (single-item and multi-item measures) did not utilize Schwartz's own measures. Thus, it is necessary to relate these two sets of data prior to performing the regression-based tests of our hypotheses. We do this in Web Appendix C, using (1) definitions and adjectives used by the BAV, Schwartz (1992, 2004), and Schwartz and Bardi (2001); (2) conceptual linkages made by others in the prior literature; (3) linguistic searches of dictionaries and thesauri; and (4) primary data from consumers through four "matching studies."

We conducted these matching studies to serve as supplementary data, aimed at uncovering the pattern of matching relationships as perceived by consumers. In these four studies, we directly asked respondents to "match" Schwartz's ten value types to our BAV image attributes after presenting descriptions of each (as depicted in columns 1 and 2 of Table 3). Participants 
Figure 1

SCHEMATIC OF DATA TRANSFORMATIONS: RATIONALE AND PROCEDURE

\begin{tabular}{|c|c|c|c|c|}
\hline Step & $\begin{array}{l}\text { Description } \\
\text { of Data }\end{array}$ & $\begin{array}{l}\text { Problem to } \mathrm{Be} \\
\text { Addressed }\end{array}$ & $\begin{array}{l}\text { Resulting Transformation } \\
\text { Applied, Logic }\end{array}$ & $\begin{array}{l}\text { Subsequent } \\
\text { Data Analysis } \\
\text { Procedures }\end{array}$ \\
\hline 1 & $\begin{array}{l}\text { Individual-level binary } \\
\text { data (yes/no) on } \\
\text { individual image } \\
\text { attributes. }\end{array}$ & $\begin{array}{l}\text { Such binary data are not } \\
\text { suitable for factor } \\
\text { analyses, and } \\
\text { hypotheses are at brand } \\
\text { (not individual) level. }\end{array}$ & $\begin{array}{l}\text { Compute brand-level } \\
\text { proportions per country for } \\
\text { each image attribute. }\end{array}$ & \\
\hline 2 & $\begin{array}{l}\text { Brand-level } \\
\text { proportions within } \\
\text { each country } \\
\text { (precentage } \\
\text { responding "yes" for } \\
\text { that image attribute for } \\
\text { that country, per } \\
\text { brand). }\end{array}$ & & & \\
\hline 3 & & & & $\begin{array}{l}\text { Factor analyses } \\
\text { (EFA, CFA) use } \\
\text { brand-level } \\
\text { proportions. }\end{array}$ \\
\hline 4 & $\begin{array}{l}\text { Brand-level } \\
\text { proportions within } \\
\text { country on individual } \\
\text { image attributes. }\end{array}$ & $\begin{array}{c}\text { Idiosyncratic response } \\
\text { style biases within each } \\
\text { country lead to variation } \\
\text { in means and standard } \\
\text { deviations for these } \\
\text { proportions across } \\
\text { countries. }\end{array}$ & $\begin{array}{l}\text { Standardize the brand-level } \\
\text { proportions within country, } \\
\text { using the means and } \\
\text { standard deviations for that } \\
\text { country on that image } \\
\text { attribute, across all brands } \\
\text { there. }\end{array}$ & \\
\hline 5 & & & & $\begin{array}{l}\text { Regressions use } \\
\text { within-country } \\
\text { standardized brand- } \\
\text { level proportions in } \\
\text { calculations of } \\
\text { standard deviation. }\end{array}$ \\
\hline
\end{tabular}

Notes: In reviewing the statistical properties of different types of ipsatization procedures, Fischer (2004) concludes that ipsatization can yield spurious factors in factor analyses (p. 273) but that regression analyses using data ipsatized (standardized) on within-group or within-culture adjustments (vs. within-subject adjustments) yield meaningful estimates of effects at individual and group/culture levels (p. 277). Thus, this is the type of within-country ipsatization we utilize. Web Appendix A provides details.

indicated the best match or selected as many matches as they liked (including a "none of these is a good match" option). The first two studies used English-speaking students from a Midwestern U.S. university (Study 1: $\mathrm{n}=115$
Study 2: $\mathrm{n}=122$ ). The other two studies (Study 3: $\mathrm{n}=258$; Study 4: $n=198$ ) used an international online panel from countries that were among (or geographically close to) the countries in our BAV data set. Those matching relationships 
Table 2

CFA: CONVERGENT AND DISCRIMINANT VALIDITY STATISTICS FOR FACTOR CONSTRUCTS

\begin{tabular}{|c|c|c|c|c|c|c|}
\hline & DV Brand Attitudes & F-Momentum & F-Elite Style & F-Friendly & F-Rugged & F-Helpful \\
\hline $\begin{array}{l}\text { DV (Brand Attitudes) } \\
\text { Alpha }=.88 \text { (best brand, high performance, high quality, } \\
\quad \text { reliable, trustworthy, worth more, leader) }\end{array}$ & .52 & & & & & \\
\hline $\begin{array}{l}\text { F-Momentum } \\
\text { Alpha }=.90 \text { (daring, dynamic, energetic, gaining in } \\
\text { popularity, innovative, progressive, up to date) }\end{array}$ & .29 & .55 & & & & \\
\hline $\begin{array}{l}\text { F-Elite Style } \\
\quad \text { Alpha }=.87 \text { (glamorous, prestigious, stylish, upper class) }\end{array}$ & .30 & .17 & .62 & & & \\
\hline $\begin{array}{l}\text { F-Friendly } \\
\quad \text { Alpha }=.70 \text { (friendly, carefree) }\end{array}$ & .34 & .41 & .08 & .56 & & \\
\hline $\begin{array}{l}\text { F-Rugged } \\
\quad \text { Alpha }=.71 \text { (rugged, tough) }\end{array}$ & .01 & .04 & .01 & .29 & .56 & \\
\hline $\begin{array}{l}\text { F-Helpful } \\
\text { Alpha }=.82 \text { (helpful, socially responsible, cares about } \\
\quad \text { customers, obliging) }\end{array}$ & .29 & .29 & .17 & .20 & .01 & .54 \\
\hline
\end{tabular}

that were consistently and unambiguously 8 indicated as the highest proportion in these data-as established through likelihood ratio tests (see Table C-1 in Web Appendix C)were taken as representing a strong perceived match between BAV attributes and Schwartz value types (column 5 in Table 3).

We then used the findings of the empirical matching studies, along with additional semantic and conceptual analyses (steps 1-3 as noted previously), to determine the final linkages between Schwartz value types and BAV items. For instance, in describing the POWER value type, Schwartz and Bardi (2001) themselves use words such as "social status," "prestige," and "public image," as well as "social power" and "authority." From the prior literature, Aaker, Benet-Martínez, and Garolera (2001, pp. 494-95) argue that brand image attributes of being upper class and glamorous should relate well to values of status and prestige, which Schwartz includes within his POWER value type. Torelli and Shavitt (2010, p. 704) note that Schwartz's conceptualization of POWER is closely linked to authoritarianism. Common adjectives for "power" include "domination," "might," "force," "strength," "privilege," "supremacy," and "unapproachable." Our supplementary empirical matching studies showed support for a match between Schwartz's POWER value and the BAV image items/factors of arrogant, unapproachable, F-Elite Style (glamorous, prestigious, stylish, upper class), F-Momentum (gaining in popularity), and F-Rugged (rugged, tough).

Utilizing all the conceptual, semantic and empirical information, the last column of Table 3 summarizes the linkages we make between the Schwartz value types and the BAV image attribute data. For the reasons detailed in Web Appendix C, and summarized in Table 3, HEDONISM is captured best

8When an image adjective cross-loaded highly on more than one Schwartz value, it was excluded from further consideration as an indicator of those Schwartz values, just as an item cross-loading highly on two factors in a factor analysis would not be considered a valid indicator of either of those factors. For example, the BAV image attribute "fun" loaded highly on both the STIMULATION and HEDONISM Schwartz values (e.g., at 54\%-55\% levels on both, in our empirical Study 4), and therefore we did not use it as an image attribute indicator for either of those values. by "sensuous" and "healthy"; POWER by "arrogant," "unapproachable," F-Elite style, F-Momentum, and F-Rugged; CONFORMITY by "restrained"; TRADITIONAL by "traditional"; and STIMULATION through the energetic component of F-Momentum. SECURITY and ACHIEVEMENT do not find good matches in the BAV data. SELF-DIRECTION is best captured through "independent," "unique," and "original," while UNIVERSALISM and BENEVOLENCE (which show high overlap) are best matched with the BAV adjectives "down to earth" and "social" as well as F-Helpful (including obliging) and F-Friendly, respectively.

Thus, our subsequent tests of $\mathrm{H}_{1 \mathrm{a}}$ use the listed BAV attributes linked to the Schwartz value types of BENEVOLENCE, UNIVERSALISM, and SELF-DIRECTION (collectively, BUS), omitting ACHIEVEMENT, which lacks good matches; our tests of $\mathrm{H}_{1 \mathrm{~b}}$ use the BAV attributes linked to POWER and HEDONISM (collectively, PH).

\section{Competitive Set Consistency: Hellinger Indices}

To enable us to explore the moderating influence of competitive context, each brand requires a measure of consistency for the set of competitors it faces in its category, taken across all its markets. For each category in each country, the BAV data set includes consumer-provided ratings for the many (but not all) competitive brands in that category, including local ones, that appeared in the BAV questionnaire. Using the list of competitive brands in each country and category, one can compute an index of competitive set consistency, called the Hellinger index (HI), fully detailed in Web Appendix D. This index measures, for a focal brand, the consistency of the competition encountered across countries in two steps. First, for each brand we computed the Hellinger affinity, a measure (see Lee 1999) that quantifies the degree to which that brand's competitors overlap in exactly two target countries. We measured this through the "usage" variable - the proportion of people who use each brand (not only the focal one) in those two countries. Second, we combine the Hellinger affinity scores for the focal brand from these multiple countries into a weighted average, the HI, with the weights being the gross domestic product of each country (adjusted for purchasing power parity). (A detailed 


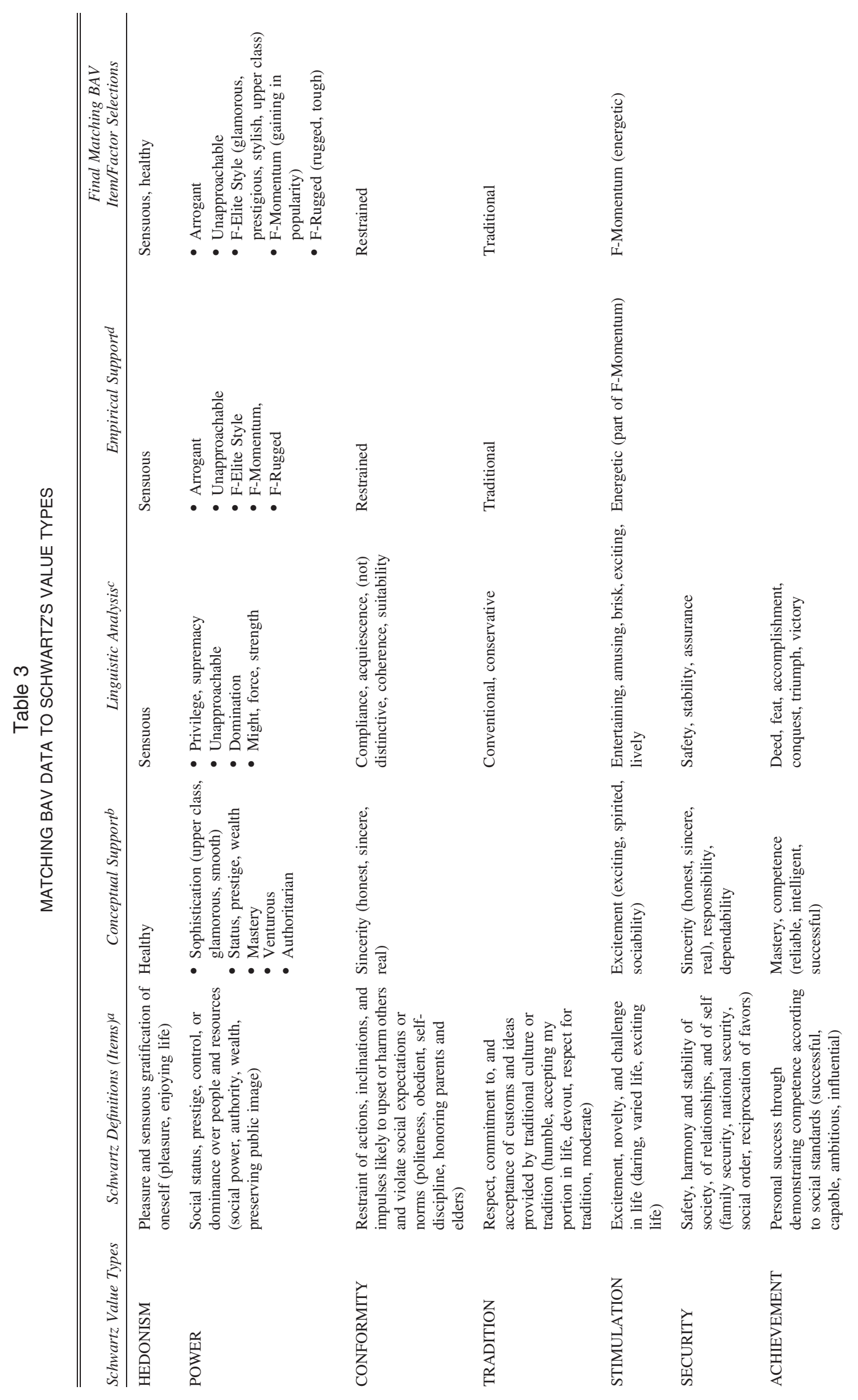




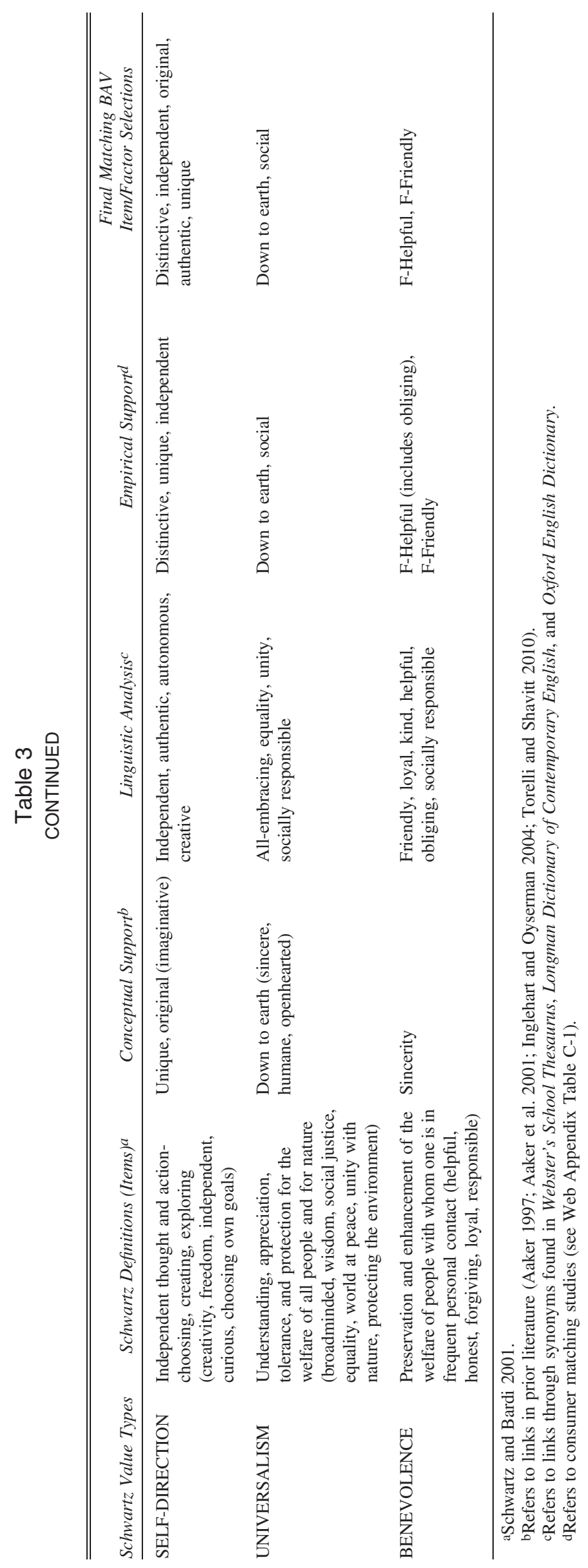


example appears in Web Appendix D, and all calculations are available in supplemental spreadsheet D-1.) In short, a higher HI score thus means greater competitive set similarity, taking into account differences in consumer usage and market size across the countries in question. For our previous examples of Leica and BNP, the HI for Leica was .73, showing greater competitive set similarity than the .13 for BNP.

\section{ANALYSES AND RESULTS}

Tests of Hypotheses

Overview. We first examine the overall expectations of $\mathrm{H}_{1}$, after which we present the tests for the specific predictions of $\mathrm{H}_{1 \mathrm{a}}$ and $\mathrm{H}_{1 \mathrm{~b}}$ (i.e., of consistent positioning on BUS vs. PH image attributes). These formal statistical tests are followed by a descriptive brand attitude analysis. We then provide a brief summary of our exploratory analyses concerning the moderating influence of competitive set consistency on the relationships in $\mathrm{H}_{1}$.

Tests of $H_{1}$ : effect of image consistency on brand attitudes. These tests employed a series of regression analyses. The dependent variable in all was the brand attitudes composite described previously (of seven performance-related attributes; e.g., best brand), standardized within country. This overall brand attitude score was regressed on (1) the cross-country consistency (SDs) measures of 15 BAV brand image single items (e.g., arrogant, unique) and five multi-item scales (e.g., F-Elite Style, F-Rugged) that had all shown adequately high levels of matching with the Schwartz value types, (2) the HI of competitive set consistency, (3) the three BAV control variables (knowledge, esteem, and relevance), and (4) a variable to capture the mean levels of the Schwartz value types to partially control for the potential confounding of means and SDs mentioned in footnote $4 .{ }^{9}$ See Table 4.

The regression estimates we relied on in Table 4 were obtained by using weighted ordinary least squares (WLS) regression, with the weights being the number of countries in which the particular brand appears (ranging from 2 to 22). In addition, because each of our brands lies (for analytical purposes) in a single product category, and because the effects of interest will likely vary across categories, we repeated the $\mathrm{H}_{1}$ analyses using a (Bayesian) hierarchical linear models (HLM) approach to account for such potential "category heterogeneity" effects. For brevity, because the HLM results were substantively very similar to the WLS

${ }^{9}$ As mentioned in footnote 4 , value types that are most consistently preferred, such as BENEVOLENCE, are also rated highly on average importance levels. Thus, the consistency with which brands communicate certain value types is necessarily confounded with the mean importance of those same values. To partially control for this potential confound, we therefore include in our analyses the first principal factor for the importance means of these values, as measured through the matched BAV attribute data. That is, for each brand, just as we computed its SD across countries on each attribute proportion, we also computed its mean across countries on each attribute. We then entered these attribute means (on each of the BAV single items and multi-item factors) into a principal components factor analysis, with brands as the unit of observation (the rows of the data matrix). The first principal factor alone accounted for approximately one-quarter of the variance in the entire set of means, further underscoring the degree of collinearity. The factor score on the first principal factor for that brand was then calculated and retained as another brand-level measure. The included principal factor improves fit slightly: weighted least squares analysis without this factor achieves an adjusted $\mathrm{R}^{2}$ of .78 ; including it raises it to .80 (d.f. $=$ 1,697). Note that all these models also include the three BAV control variables. results, we do not discuss the HLM results in the text; Table 4 presents both sets of results for easy comparison. Web Appendix E describes the HLM technique, the models we estimated using it, our HLM results, and our commentary on those results.

Omnibus test of $H_{1}$. It is important to first determine whether the overall pattern of all the attribute-linked regression coefficients in Table 4 is consistent with our core theory-based expectations $\left(\mathrm{H}_{1}\right)$. We have argued that brands should benefit (vs. lose) from being consistently positioned across markets on image attributes that match consumer values that are themselves consistently important (vs. vary in importance). This implies that inconsistency on a brand image variable (higher SD) should yield positive impact on brand attitudes, reflected by a more positive regression coefficient, when the image attribute corresponds to a value that has higher cross-cultural variation in importance (a higher Schwartz SD). Analogously, greater inconsistency (higher $\mathrm{SD}$ ) on an image attribute should yield negative (or less positive) regression coefficients when the image attribute corresponds to a Schwartz value with lower cross-cultural importance variation (a lower Schwartz SD). In summary, our hypothesis predicts a positive correlation between the Schwartz SDs of the matching values and our regression coefficients.

To test this omnibus expectation, we computed the correlation between the WLS regression coefficients estimated and their corresponding Schwartz SDs across our 20 brand image variables/factors listed as independent variables in Table 4. This correlation coefficient between the Schwartz SDs and the WLS regression coefficients is $.60(p<.005) .{ }^{10}$ To check the robustness of this analysis to scaling assumptions, we repeated it by retaining only the signs of the regression coefficients and estimating a binary logistic regression with the Schwartz means' principal factor as the sole covariate. Results were substantively identical $\left(\chi^{2}(1)=6.9, p<.01\right){ }^{11}$ Thus, our core theoretical expectation in $\mathrm{H}_{1}$ finds support at this overall level.

We next turn to the individual regression coefficients in Table 4 to determine whether they support the directional subhypotheses $\mathrm{H}_{1 \mathrm{a}}$ and $\mathrm{H}_{1 \mathrm{~b}}$. We test for such support at two levels: at the most disaggregate level (of individual variables/ factors) and at a more aggregate level (with relevant variables/ factors pooled together).

Disaggregate test of $H_{1 a}$ : negatively significant relations. Table 4 contains our disaggregated regression coefficients, with the variables and factors ordered to match the following results presentation. We first use the WLS estimates to test $\mathrm{H}_{1 \mathrm{a}}$, the hypothesis that multicountry brands should have higher overall brand attitudes if they are more consistently positioned across their markets (with smaller cross-country SDs) on image attributes that match the Schwartz values that are desired most similarly across our BAV markets (i.e., BUS). Thus, we hypothesize a negative relationship for these three value types, such that a higher SD (more inconsistency) should reduce

10 Note that the $r=.60$ value translates into a $t=.6 / \operatorname{sqrt}\left[\left(1-.6^{2}\right) /(n-2)\right]$, where $\mathrm{n}=20$, so $\mathrm{t}=3.182$ (d.f. $=18$ ), implying a $p$-value of approximately .0026 .

${ }^{11}$ Note that we included the full set of regressors in these two prior analyses, as theory dictates, but did not systematically account for their varying significance levels. To guard against this, we next took 1 million samples from the joint posterior distribution of all the (standardized, WLS) regression coefficients and computed $\mathrm{r}$ and $p$-values for each. The median $\mathrm{r}$ is .573, median $p$-value is .0078, and the histogram of $r$ (not included, for brevity) is extremely strongly bounded away from zero. (Details of all these tests appear in Web Appendix E.). 
Table 4

TEST OF $\mathrm{H}_{1}$ : WLS AND HLM ESTIMATES (STANDARDIZED COEFFICIENTS)

\begin{tabular}{|c|c|c|c|}
\hline \multirow[b]{2}{*}{ Variable } & \multirow[b]{2}{*}{$\begin{array}{c}\text { WLS } \\
\text { Fixed Effects }\end{array}$} & \multicolumn{2}{|c|}{$H L M$} \\
\hline & & Fixed Effects & $\begin{array}{l}\text { Random Effects: } \\
\text { Variances }\end{array}$ \\
\hline Intercept & $-4.307 * *(.149)$ & $-3.790 * *(.169)$ & $.919(.020)$ \\
\hline $\mathrm{HI}$ & $.658 * *(.053)$ & $.681 * *(.065)$ & $.398(.075)$ \\
\hline Arrogant & $.040^{*} \quad(.025)$ & $.044 \quad(.031)$ & $.043(.018)$ \\
\hline Authentic & $-.041 \quad(.026)$ & $-.011 \quad(.028)$ & $.070(.031)$ \\
\hline Distinctive & $.025 \quad(.025)$ & $.029 \quad(.027)$ & $.025(.025)$ \\
\hline Down to earth & $.062 * *(.026)$ & $.049 * \quad(.029)$ & $.062(.016)$ \\
\hline Fun & $-.357 * *(.030)$ & $-.308 * *(.035)$ & $.092(.034)$ \\
\hline Healthy & $.141 * *(.022)$ & $.088 * *(.028)$ & $.118(.026)$ \\
\hline Independent & $.018 \quad(.023)$ & $-.005 \quad(.028)$ & $.127(.020)$ \\
\hline Original & $-.054 * *(.025)$ & $-.035 \quad(.030)$ & $.095(.017)$ \\
\hline Restrained & $.010 \quad(.022)$ & $.020 \quad(.026)$ & $.066(.018)$ \\
\hline Sensuous & $-.039 * \quad(.022)$ & $-.012 \quad(.025)$ & $.009(.013)$ \\
\hline Social & $-.089 * *(.026)$ & $-.119 * *(.029)$ & $.046(.023)$ \\
\hline Traditional & $-.043^{*} \quad(.025)$ & $-.001 \quad(.030)$ & $.102(.026)$ \\
\hline Trendy & $.000 \quad(.026)$ & $-.005 \quad(.028)$ & $.071(.014)$ \\
\hline Unapproachable & $.102 * *(.023)$ & $.064 * *(.027)$ & $.046(.012)$ \\
\hline Unique & $-.042 * \quad(.024)$ & $-.039 \quad(.026)$ & $.052(.016)$ \\
\hline F-Elite Style & $.137 * *(.034)$ & $.154 * *(.039)$ & $.090(.048)$ \\
\hline F-Friendly & $-.082 * *(.032)$ & $-.078 * *(.035)$ & $.049(.024)$ \\
\hline F-Helpful & $.112 * *(.036)$ & $.144 * *(.040)$ & $.102(.039)$ \\
\hline F-Momentum & $.174 * *(.041)$ & $.118 * *(.048)$ & $.037(.055)$ \\
\hline F-Rugged & $.204 * *(.027)$ & $.192 * *(.031)$ & $.100(.022)$ \\
\hline $\begin{array}{l}\text { First factor of } \\
\text { means }\end{array}$ & $.219 * *(.020)$ & $.251 * *(.021)$ & $.004(.006)$ \\
\hline Knowledge & $-.112 * *(.015)$ & $-.106 * *(.017)$ & $.024(.001)$ \\
\hline Esteem & $1.371 * *(.046)$ & $1.216^{* *}(.057)$ & $.015(.001)$ \\
\hline Relevance & $-.509 * *(.030)$ & $-.473 * *(.035)$ & $.018(.001)$ \\
\hline
\end{tabular}

attitudes. Of the nine variables/factors that represent these three BUS Schwartz value types (F-Friendly, F-Helpful, social, downto-earth, distinctive, authentic, original, unique, and independent), three are significant (F-Friendly, social, and original; $p<.05$ ) and one is marginally significant (unique; $p<.09$ ) in the predicted negative direction. Two (down-to-earth and F-Helpful) are significant in the opposite (positive) direction. Three others (authentic, independent, distinctive) are nonsignificant. Thus, we do not find support for $\mathrm{H}_{1 \mathrm{a}}$ in Table 4 at this most disaggregate level (only three of nine variables support it).

Disaggregate test of $H_{1 b}$ : positively significant relations. $\mathrm{H}_{1 \mathrm{~b}}$, however, does find substantial support even at this most disaggregate level (in Table 4). This hypothesis argued that brands that are more consistent across their markets (smaller SDs), on those image attributes that vary the most in desirability across markets per Schwartz's values typology $(\mathrm{PH})$, should have lower overall brand attitudes. Accordingly, we hypothesize a positive relationship, such that a higher SD (more inconsistency) should increase brand attitudes.

Of the seven variables/factors that represent these two $\mathrm{PH}$ Schwartz value types (sensuous, healthy, arrogant, unapproachable, F-Elite Style, F-Momentum/gaining in popularity, F-Rugged), five are significant at $p<.05$ in the predicted positive direction (healthy, unapproachable, F-Elite Style, F-Momentum, and F-Rugged). One attribute approaches significance (arrogant; $p<.10)$ in the predicted positive direction, and one approaches significance in the opposite direction (sensuous; $p<.08$ ). Thus, five of seven coefficients support $\mathrm{H}_{1 \mathrm{~b}}$. In addition, though this finding is not part of a hypothesis test, we note that of the other BAV items in the regression estimates that operationalize the Schwartz value types that appear in the middle of the SDs range, only one is marginally significant, in a negative direction (traditional; $p<.08$ ).

Test of $H_{1 a}$ and $H_{1 b}$ at a pooled level. We also performed a test of these directional subhypotheses at a pooled level, estimating a second WLS regression model in which we used the SDs of only the individual image factors/variables that are relevant to $\mathrm{H}_{1 \mathrm{a}}$ and $\mathrm{H}_{1 \mathrm{~b}}$, aggregating these into two composites, SD_BUS and SD_PH. We computed SD_BUS using the square root of the sum of the variances of its nine components, and SD_PH as the square root of the sum of the variances of its seven components (all listed previously with the disaggregate tests). Such aggregation of the image factors and variables should increase the power of our test (Gelman and Hill 2006, chapter 4.6), albeit at the expense of a disaggregate account.

The same control variables listed in Table 4 were used in this second WLS regression model (knowledge, esteem, and relevance; the first factor of means; and the HI), and all independent variables were mean-centered. In this model estimate, SD_BUS had a significant negative effect on brand attitudes $(-.047, p<$ .001 ), indicating that a higher SD (i.e., reduced consistency) of the BUS composite relates negatively to brand attitudes, in support of $\mathrm{H}_{1 \mathrm{a}}$. However, SD_PH had a significant positive effect on brand attitudes $(.129, p<.001)$, indicating that a higher $\mathrm{SD}$ (reduced consistency) on the $\mathrm{PH}$ attributes relates positively to brand attitudes, in support of $\mathrm{H}_{1 \mathrm{~b}}$. Thus, these pooled analyses show support for both $\mathrm{H}_{1 \mathrm{a}}$ and $\mathrm{H}_{1 \mathrm{~b}}$.

Discussion of $H_{1}$ results. Thus, summarizing these results, we find that the attitudinal payoff for brands that pursue a highconsistency strategy in their positioning worldwide does indeed depend on whether the specific image attributes on which they are consistently positioned are themselves desired uniformly across those markets (support for $\mathrm{H}_{1}$ in the omnibus test). For our directional subhypotheses, the empirical evidence is stronger for $\mathrm{H}_{1 \mathrm{~b}}$ - supported at both the pooled and disaggregate levels of analysis - showing that if those image attributes represent values that vary most in their global appeal (e.g., PH), it is wiser for multicountry brands to vary their use rather than deploy them consistently.

The empirical evidence in our data are somewhat less supportive for the relationship in the opposite direction $\left(\mathrm{H}_{1 \mathrm{a}}\right)$, which argued that for brand image attributes that represent values that vary less in their global appeal (e.g., BUS), global brands are better served by a strategy of consistent worldwide positioning. For $\mathrm{H}_{1 \mathrm{a}}$, we do not find support at the most disaggregate level, but support does exist at the pooled level. In our "Discussion" section, we review several possibilities for why we find a relatively weaker level of support of $\mathrm{H}_{1 \mathrm{a}}$.

\section{Descriptive Brand Attitude Analysis of the Danger from PH Consistency}

The significant results for $\mathrm{H}_{1 \mathrm{~b}}$ suggest that brands positioned consistently across countries on $\mathrm{PH}$ values run the real risk of generating less positive attitudes in countries where these two values are not rated as important. (In our BAV data, for instance, $\mathrm{PH}$ values have their relatively highest mean importance ratings 
in China and Japan, and their lowest ratings in Chile and Italy). We tested this implication directly, again at the pooled PH levels for greater power and ease of analysis. First, we calculated the means of each brand on the pooled PH attributes for each country. We then calculated each brand's cross-country SD (consistency) on these pooled PH attributes. Next, we conducted a median split on these brand-level PH SDs to find the 50\% of the brands (halfsample $\mathrm{n}=860$ brands) that were relatively lower (vs. higher) in their cross-country SDs (consistency) on these PH attributes.

We then tested whether, for this half-sample of brands positioned more consistently across countries on PH values (i.e., in the lower half of the PH SDs), such uniform positioning helped or hurt them in countries that were either relatively high or low on the importance they gave to $\mathrm{PH}$ values. To test this, we regressed our brand attitude dependent variable (1) on the importance ratings for the combined $\mathrm{PH}$ values in that country (dummycoded as $1=$ above-average importance and $0=$ below-average importance, using country-level data that we obtained from Shalom Schwartz) and (2) using the brand's mean level on pooled $\mathrm{PH}$ attributes across these countries as a covariate to control for the possible SD-mean confound (as in our Table 4 test).

This regression (using ordinary least squares, because it is on a country level and not a brand level) showed a significant positive coefficient for the country-level PH importance dummy variable $(\mathrm{b}=.040, p<.037)$ on that brand's attitudinal ratings, with the significant positive coefficient indicating a higher brand attitude in countries that value $\mathrm{PH}$ versus not. However, this difference in brand attitudes across countries where $\mathrm{PH}$ values are highly rated (vs. not) is significant only for PH-consistent brands $\left(\mathrm{M}_{\text {High_PH_Imp }}=.098, \mathrm{M}_{\text {Low PH_Imp }}=.059 ; p<.043\right)$; it is insignificant for PH-inconsistent brands $\left(\mathrm{M}_{\mathrm{High} \_P H} \_\mathrm{Imp}=.05\right.$, $\mathrm{M}_{\text {Low_PH_Imp }}=.028 ; p>.2$ ). Thus, brands that are positioned more uniformly on $\mathrm{PH}$ values have statistically higher consumer attitudinal ratings in countries that themselves give greater importance to $\mathrm{PH}$ values than in countries that give lower importance to them, but brands positioned less uniformly on PHvalues do not have higher attitudinal ratings. This result shows the significant attitudinal implications of $\mathrm{H}_{1 \mathrm{~b}}$; we present some examples from our BAV data in the "Discussion" section.

We then conducted a similar regression analysis for the $50 \%$ of brands positioned more consistently on combined BUS attributes $\left(\mathrm{H}_{1 \mathrm{a}}\right)$. Recall that the theory predicts that BUS values should be rated uniformly high in importance across most countries, whereas $\mathrm{PH}$ values should show greater variation across countries in these importance ratings. Thus, we should see a greater range (variance) in cross-country importance ratings for $\mathrm{PH}$ versus BUS, and this is borne out in our sample of test countries $(\mathrm{PH}$ range [variance] $=1.06$ [.0676]; BUS range $[$ variance] $=.68[.0361] ; p<.04)$. With the smaller variance in BUS importance ratings across our test countries, the median split technique produces countries that value BUS only slightly less than others and so provides less meaningful variation for carrying out the analysis. As a result, the analogous country dummy variable for BUS in this regression test (for high vs. low BUS importance) was not significant ( $b=$ $.001, p=.972$ ). Thus, again, the attitudinal implications of $\mathrm{H}_{1 \mathrm{~b}}$ have more statistical support than those for $\mathrm{H}_{1 \mathrm{a}}$.

\section{Exploratory Test of the Moderating Influence of Competitive} Set Consistency

As we stated in our conceptual development, we also aim to explore how the attitudinal effects of a brand's consistency of image positioning across countries may change with varying levels of competitive set consistency (measured by the HI, described previously). Specifically, for the reasons we have provided, we expect that if the brand faces a more heterogeneous set of competitors across its markets (lower HI), greater overall image consistency by the focal brand should relate negatively to overall consumer attitudes.

Because this expectation is stated without reference to any specific Schwartz value types, we explored this in terms of a brand's overall image positioning consistency on all $41 \mathrm{BAV}$ image attributes (i.e., all except the ones used to measure brand attitudes). We estimated a WLS regression model with the same attitudinal dependent variable as in our $\mathrm{H}_{1}$ tests, and four independent variables: the total brand image positioning consistency measure (SD41), the HI, the interaction term between the two, and the scores on the first factor for the means on all 41 image attribute items (as a control). These WLS results (detailed in Web Appendix F) showed that the interaction between the HI and the SD41 index was significant in a negative direction. A plot of this interaction (in Web Appendix F) shows that higher overall image consistency by the brand (a lower SD41) does relate more positively to consumer attitudes if the brand faces a homogeneous (vs. heterogeneous) set of competitors (higher HI), consistent with the prior theorizing of Jain (1989), Samiee and Roth (1992), and Zou and Cavusgil (2002).

Might the influence of competitive context on the effectiveness of standardization further depend on whether the standardization (brand positioning consistency) under consideration is on the uniformly important BUS attributes versus on the less uniformly important $\mathrm{PH}$ attributes? To explore this possibility, we repeated the WLS and interaction plots analyses reported previously, but instead of using our overall image consistency SD41 variable, we used separate measures of the brand's consistency on the BUS and PH image attributes (SD-BUS and SD-PH). Recall that we had found that a consistently positioned multicountry brand's attitudes become lower when facing heterogeneous (vs. homogeneous) competitors across its various markets. Our exploratory analysis showed that this decrease in attitudes is larger if the brand is more (vs. less) consistent in its own BUS attributes positioning as well as if the brand is less (vs. more) consistent in its own positioning on PH attributes. Web Appendix F presents details of these exploratory analyses (WLS and descriptive plots) as well as interpretive commentary.

\section{DISCUSSION}

\section{Results and Theoretical Contributions}

Our results make multiple contributions to the literature on cross-cultural values and the theory of global brand management. First, through our integration and analysis of the literature on cross-cultural values, we identify which specific values are considered uniformly more important across more cultures, and for what functional and evolutionary reasons. Second, we apply the conclusions of this cross-cultural values analysis to the substantive domain of global brand management, proposing and showing the differential impact on attitudes toward a global brand if it is positioned consistently on consumer needs and values that are more similar (vs. varied) in their importance across multiple countries. We thus add to the work by Torelli et al. (2012). We also explore for the first time the moderating influence of competitive set heterogeneity on the effectiveness of standardized global positioning strategy. 
Contribution to the cross-cultural values literature. Our use of life values allowed us to tap into the rich theoretical and empirical streams of prior work on the existence and evolution of the adaptive societal functions of cultural values (Parsons 1951; Schwartz 1992, 2004). This gave us a strong basis for developing expectations regarding the specific values for which there is more (vs. less) cross-cultural variation in importance (Schwartz and Bardi 2001) and for what theoretical reason (the shifting societal priorities served by these specific cultural values; Gelfand et al. 2011; Henrich 2004; Inglehart and Oyserman 2004; Norenzayan and Shariff 2008; Norenzayan et al. 2016). Through this integration and analysis of the literature on how societies evolve in the relative importance they place on specific cultural values over time, we show why there is relatively more equality in the cross-national importance of BUS values (i.e., BENEVOLENCE, UNIVERSALISM, and SELFDIRECTION) and less equality in importance for PH values (i.e., POWER and HEDONISM). This theoretically developed specific identification makes an important contribution to the literature on cross-national cultural differences.

Standardized versus localized positioning strategies for global brands. We then apply our theoretical conclusions to international marketing strategy. The extant view in this literature is that there is typically a trade-off between the opposing advantages of standardization and localization in brand positioning, and that standardization makes sense only if there is enough homogeneity in consumer wants and needs relative to those standardized elements (Jain 1989; Samiee and Roth 1992; Zou and Cavusgil 2002). However, as Ryans, Griffith, and White (2003) point out, this literature typically treats the presence or absence of adequate homogenization of consumer needs in a binary fashion rather than examining the extent of homogenization of markets on these different needs; nor does it provide a theory-based articulation of which specific brand positioning attributes or messages face relatively more homogeneity (vs. heterogeneity) in consumer response across markets. We address both these gaps in this article.

We then suggest and show that global brands can do a better job in managing this trade-off-between the cost and launchspeed advantages of standardization versus the need for localized relevance-if they strategically standardize less on those specific image attributes with significant cross-market variation in importance ratings. To do this, we use new conceptual and empirical analyses to match specific BAV data image attributes to these underlying life values to test our hypothesized relationships within our BAV data set. Thus, we argue that multicountry brands in our data set that are positioned consistently on BAV image attributes reflecting BUS values gain in brand attitudes (e.g., friendly, social, original), whereas those positioned consistently on PH values suffer (e.g., unapproachable, momentum, rugged). Importantly, instead of treating consistent versus inconsistent positioning on these image attributes as a binary either/ or choice, we quantify the degree of positioning consistency on these attributes, meeting a documented gap in the literature (Ryans, Griffith, and White 2003) and drawing conclusions that managers can implement (by adjusting the degree of crossmarket consistency for their brands, by attribute).

Our unique BAV global field data set enabled us to put this key hypothesis to an empirical test, and it found substantial support, especially for the part dealing with values that vary more (PH values). While the fact that this BAV data set was not originally designed and formulated to test our specific propositions created several challenges for our analyses, it nonetheless provided a rare opportunity to test our theorybased hypotheses through our extensive data preparation work and our supplementary "variable matching" data and analysis. Despite remaining limitations (discussed next), the fact that our data set uses perception and preference data from over 64,000 consumers, in 22 widely dispersed countries, on 1,723 multicountry brands in 27 product categories, adds considerable empirical weight to these findings. Thus, our results add a vital new qualification to the common managerial belief that global brands marketed in a more consistent, standardized way also ought to reap the benefits of higher consumer attitudes (in addition to possible cost efficiencies). We show that this is less likely if the specific type of brand attribute on which the brand is more consistently positioned is the kind of attribute that consumers across the world desire less uniformly (PH values).

Interestingly, the evidence for an increase in brand attitudes from consistent positioning on image attributes that are uniformly valued everywhere (BUS values) is somewhat less strong. Several possibilities can explain this finding. First, it may indeed be the case that $\mathrm{H}_{1 \mathrm{a}}$ does not uniformly apply for all the value types within it, but rather only for some (e.g., more for BENEVOLENCE and UNIVERSALISM than SELFDIRECTION). Second, it may be that even if brands are less consistent globally in their deployment of BUS-related appeals, they can still find other local positioning platforms that resonate with local consumers so that their inconsistent use of BUS positioning does not end up hurting them attitudinally in our data. Third, it could be that if many brands choose to compete on these consistently highly valued image attributes, a brand positioning platform that relies on these attributes may simply be less differentiating or compelling. Just as consumers consider brand information that seems negative to be more diagnostic because it is less common (compared with positive information) and thus overweigh it in their brand judgments (Skowronski and Carlston 1989), it may be that equally liked but commonly used BUS attributes may become less important in consumer judgments.

Fourth, it may be that for certain types of brands that pursue a segmented (vs. mass-market) strategy, greater market success may accrue from positioning their brand on narrower, non-BUS segment-relevant appeals rather than following a BUS positioning that may be optimal for a broad market. In particular, because luxury brands usually appeal to consumers who care more about "self-enhancement" (dominance over other people, status, power, etc.) than about "self-transcendence" (helping other people and society rather than oneself), globally consistent positioning on $\mathrm{PH}$ could logically be a more successful strategy for luxury brands than positioning on BUS (Torelli, Monga, and Kaikati 2012). Future research is needed to explore all these possibilities.

Our other contribution concerns the impact of a varying competitive environment, which has not yet been empirically examined in the global marketing literature. Using the HI measure of competitive set similarity, we provide the first direct evidence that a global brand facing dissimilar major competitors in its many markets benefits more from responsively customizing (localizing) its competitive positioning, rather than pursuing the same one. Although our measures utilize only the degree of consistency in the focal brand's positioning across markets (and not the strength of this positioning), we find that 
when facing heterogeneous competitors across many markets, brand attitudes are higher if the focal brand's own positioning consistency is low rather than high. Furthermore, when the competitive context is more homogeneous, brands benefit from their own image consistency more on BUS than on PH values. Our results suggest that the variable of competitive similarity deserves more attention in the international marketing strategy literature. We note here that in our BAV data it seemed that the degree of competitive set homogeneity might be a function of the nature of the industry/category - that is, there may be more variation in a service industry such as banking than in a capital-intensive durables product such as automobiles. Thus, the ability of managers to vary the degree of their overall positioning consistency, as a function of competitive set heterogeneity, may be constrained by the nature of their industry sector.

\section{Managerial Implications}

In a world that is characterized both by the sweeping forces of globalization (e.g., Alden et al. 1999) and by consumer desires for localization (e.g., Hannerz 1990), multicountry marketers must find a way to combine local appeal with global efficiency. Hollis (2008) writes, “Today's global brands must leverage their advantages of scale and adapt their offering to ensure local relevance" (p. 82). The only way to do both of these, he adds, "is to identify a promise that works across countries" (pp. 165-66). Citing Simon Clift, the chief marketing officer of Unilever, he stresses that it is much more critical to find a brand appeal that works across borders, so that brand assets can be created on a one-size-fits-all basis, than it is to use a common brand name. "A global promise is the most important global brand asset, way more important than the same name or formulation or graphics," he writes (p. 174).

Consistent with Hollis's (2008) urging, our work also shows that brands that intend to become global need to incorporate as many universally desired needs and values as possible (or at least find a key value that they can best satisfy). Finding such globally appealing brand promises requires clever consumer market research because "the real trick lies in looking for commonalities, not differences" (Hollis 2008, p. 156). Our research has focused precisely on this issue-that is, of avoiding life values that represent the differences rather than the commonalities - to facilitate the consistent cross-market positioning by global brands on image attributes that will yield economies of scale without jeopardizing local market appeal.

In this context, our finding that brand appeals to POWER and HEDONISM can vary in their cross-market payoff merits particular attention. Evidence does exist (in our BAV data and in Hollis [2008]) that national attitudes about luxury and statusor even to prestigious brands - vary enormously across countries (e.g., more favorable in Russia and China than in Western Europe, the United States, and Australia). Thus, the egalitarian "Campaign for Real Beauty" for Dove soap was much more successful in the more egalitarian set of countries than in the former countries (Hollis 2008, pp. 62, 223). (In our BAV data, Dove's attitude ratings were 1.11 in Chile, the country that gave the lowest importance to $\mathrm{PH}$, but -.05 in Japan, the country that gave the highest importance to $\mathrm{PH}$.) This example suggests that a multicountry, mass-market brand consistently positioned on status will do well in some markets but not in others, yielding scale economies of consistency but sacrificing share in some markets. Alternatively, such a mass-market brand could instead prioritize local market relevance, using a status appeal in some markets but not others. This would grow overall demand but sacrifice economies of scale from consistency.

But why choose status at all? Could the multicountry brand not position itself on a different image attribute altogether, something less divisive, to avoid being negatively affected in its search for global scale? Thus, to return to the example of Apple's Mac versus PC ad campaign: Apple's ads might have been received equally well in Japan and the United States if they had, in both countries, downplayed Mac's hedonistic benefits (of pleasure, enjoyment, etc.) — something not equally desired everywhere - and focused instead on its greater ability to facilitate self-direction (creativity and independent thought), which is more universally sought.

The findings and implications in this article thus ought to be of considerable value in the strategy development processes for global brands. Although in some cases there may be natural limits on which image attributes a multicountry brand can position itself in its many markets (as pointed out by Torelli et al. [2012]), most often brands do have the flexibility to modify their brand image positioning in different countries. McDonald's, for instance, is able to position itself on convenience and value in developed countries, while emphasizing an aspirational and upmarket brand image in many developing countries. Multicountry brands thus can undergo reinterpretation of their promises and values as they seek success in different cultures. However, they have not previously been provided evidence of how the importance of specific cross-cultural values might affect the success of their positioning decisions, as done here.

\section{Limitations and Future Research}

In this article, we were unable to systematically study the variations in results across product categories (varying on perceived risk, social signaling value, etc.), because we had no external data on these variations. Furthermore, given that we were studying 27 product categories at once, we were limited to brand image attributes as opposed to functional/utilitarian attributes; future research needs to study the latter as well. In addition, although we attempted in our regression analyses to control for the near confound of values importance crosscultural SDs and their means by using the first factor of these means, superior methods need to be found to control for this.

Endogeneity concerns also exist, because our field data necessarily result from decisions by firms rather than from an experimental design. For us to draw strategic inferences from our regression coefficients, we need to assume that the data include a mixture of smart firms that know that a consistent global image should be positioned on dimensions that consumers universally like (as we identified in $\mathrm{H}_{1}$ ) and some less astute firms that lack this knowledge or are unable to position themselves on universally liked dimensions because of constraints in information, resources, channels, and so on. This is, in our view, a reasonable set of assumptions.

The alternative causal possibility is that consumers everywhere know well the brands they like and thus rate them highly on the image dimensions they like, even if they never actually thought of the brands in terms of such imagery. Such a possibility is not supported by our data. When we calculated for each brand the SD across the mean levels of its $48 \mathrm{BAV}$ raw attribute ratings and compared these between the brands that were higher versus lower in brand attitudes (using a median split), we found no difference (.64 vs. .64, $p>.5$ ). Therefore, although we do not believe that endogeneity concerns threaten the validity of our analyses, future 
research should examine causal sequences more thoroughly (such as by using cross-lagged panel data or controlled laboratory experiments). Future studies might also delve more deeply into how societal values - and thus, a preference for more consistent multicountry brands - evolve over time. Our cross-sectional data did not allow for such analysis.

\section{REFERENCES}

Aaker, Jennifer L. (1997), "Dimensions of Brand Personality," Journal of Marketing Research, 34 (3), 347-56.

Aaker, Jennifer, Veronica Benet-Martínez, and Jordi Garolera (2001), "Consumption Symbols as Carriers of Culture: A Study of Japanese and Spanish Brand Personality Constructs," Journal of Personality \& Social Psychology, 81 (3), 492-508.

Aaker, Jennifer L., and Angela Y. Lee (2001), “'I' Seek Pleasures and 'We' Avoid Pains: The Role of Self-Regulatory Goals in Information Processing and Persuasion," Journal of Consumer Research, 28 (1), 33-49.

Alden, Dana L., Jan-Benedict E.M. Steenkamp, and Rajeev Batra (1999), "Brand Positioning Through Advertising in Asia, North America, and Europe: The Role of Global Consumer Culture," Journal of Marketing, 63 (1), 75-87.

Arnold, David J., and Hans-Willi Schroiff (2004), "Strategies for Managing Brand and Product in International Markets," in The Global Market: Developing a Strategy to Manage Across Borders, J.A. Quelch and R. Deshpande, eds. San Francisco: Jossey-Bass, 163-79.

Batra, Rajeev, Pamela Homer, and Lynn R. Kahle (2001), "Values, Susceptibility to Normative Influence, and Attribute Importance Weights: A Nomological Analysis," Journal of Consumer Psychology, 11 (2), 115-28.

Baumgartner, Hans, and Jan-Benedict E.M. Steenkamp (2001), "Response Styles in Marketing Research: A Cross-National Investigation," Journal of Marketing Research, 138 (2), 143-56.

Carpenter, Gregory S., Rashi Glazer, and Kent Nakamoto (1994), "Meaningful Brands from Meaningless Differentiation: The Dependence on Irrelevant Attributes," Journal of Marketing Research, 26 (3), 339-50.

Chandon, Pierre, and Brian Wansink (2007), "Is Obesity Caused by Calorie Underestimation? A Psychophysical Model of Meal Size Estimation," Journal of Marketing Research, 44 (1), 84-99.

Copeland, Lennie, and Lewis Griggs (1985), "Chapter 3," in Going International: How to Make Friends and Deal Effectively in the Global Marketplace. New York: Random House.

Craig, C. Samuel, and Susan P. Douglas (2000), "Configural Advantage in Global Markets," Journal of International Marketing, 8 (1), 6-25.

Dichter, Ernest (1985), "What's in an Image," Journal of Consumer Marketing, 2 (1), 75-81.

Fischer, Ronald (2004), "Standardization to Account for CrossCultural Response Bias: A Classification of Score Adjustment Procedures and Review of Literature Research in JCCP, "Journal of Cross-Cultural Psychology, 35 (3), 263-82.

Fornell, Claes, and David F. Larcker (1981), "Evaluating Structural Equation Models with Unobservable Variables and Measurement Error," Journal of Marketing Research, 18 (1), 39-50.

Gelfand, Michele J., Jana L. Raver, Lisa Nishii, Lisa M. Leslie, Janetta Lun, Beng Chong Lim, et al. (2011), "Differences Between Tight and Loose Cultures: A 33-Nation Study," Science, 332 (6033), 1100-04.

Gelman, Andrew, and Jennifer Hill (2006), Data Analysis Using Regression and Multilevel/Hierarchical Models. New York: Cambridge University Press.

Gimeno, Javier (1999), "Reciprocal Threats in Multimarket Rivalry: Staking Out 'Spheres of Influence' in the U.S. Airline Industry,' Strategic Management Journal, 20 (2), 101-28.
Gupta, Susan F., Doan Winkel, and Laura Peracchio (2009), "Cultural Value Dimensions and Brands: Can a Global Brand Image Exist?" in Handbook of Brand Relationships, J. Priester, D. MacInnis, and C.W. Park, eds. Armonk, NY: M.E. Sharpe, 230-46.

Hannerz, Ulf (1990), "Cosmopolitans and Locals in World Culture," in Global Culture: Nationalism, Globalization and Modernity, M. Featherstone, ed. Thousand Oaks, CA: Sage Publications, 295-310.

Henrich, Joseph P. (2004), "Cultural Group Selection, Coevolutionary Processes and Large-Scale Cooperation," Journal of Economic Behavior \& Organization, 53 (1), 3-35.

Hofstede, Geert H. (2001), Culture's Consequences: Comparing Values, Behaviors, Institutions, and Organizations Across Nations. Thousand Oaks, CA: Sage Publications.

Hollis, Nigel (2008), The Global Brand. New York: Palgrave Macmillan.

Inglehart, Ronald, and Daphna Oyserman (2004), "Individualism, Autonomy and Self-Expression: The Human Development Syndrome," in Comparing Cultures: Dimensions of Culture in a Comparative Perspective, H. Vinken, J. Soeters, and P. Ester, eds. Leiden, The Netherlands: Brill, 74-96.

Jain, Subhash C. (1989), "Standardization of International Marketing Strategy: Some Research Hypotheses," Journal of Marketing, 53 (1), 70-79.

Karnani, Aneel, and Birger Wernerfelt (1985), "Multiple Point Competition," Strategic Management Journal, 6 (1), 87-96.

Lee, Lillian (1999), "Measures of Distributional Similarity," in Proceedings of the 37th annual meeting of the Association for Computational Linguistics on Computational Linguistics. Stroudsburg, PA: Association for Computational Linguistics, 25-32.

Neff, Jack (1999), "P\&G and Unilever's Giant Headaches," Advertising Age, 70 (22), 22-28.

Norenzayan, Ara, and Azim F. Shariff (2008), "The Origin and Evolution of Religious Prosociality," Science, 322 (5898), 58-62.

Norenzayan, Ara, Azim F. Shariff, Will M. Gervais, Aiyana K. Willard, Rita A. McNamara, Edward Slingerland, et al. (2016), "The Cultural Evolution of Prosocial Religions," Behavioral and Brain Sciences, 39, 1-65.

Parsons, T. (1951), The Social System. New York: The Free Press. Preacher, Kristopher J., Derek D. Rucker, Robert C. MacCallum, and W. Alan Nicewander (2005), "Use of the Extreme Groups Approach: A Critical Reexamination and New Recommendations," Psychological Methods, 10 (2), 178-92.

Quelch, John A., and Edward J. Hoff (1986), "Customizing Global Marketing," Harvard Business Review, 64 (May/June), 59-68.

Ryans, John K., Jr., David A. Griffith, and D. Steven White (2003), "Standardization/Adaptation of International Marketing Strategy: Necessary Conditions for the Advancement of Knowledge," International Marketing Review, 20 (6), 588-603.

Samiee, Saeed, and Kendall Roth (1992), "The Influence of Global Marketing Standardization on Performance," Journal of Marketing, 56 (2), 1-17.

Schwartz, Shalom H. (1992), "Universals in the Content and Structure of Values: Theoretical Advances and Empirical Tests in 20 Countries," in Advances in Experimental Social Psychology, Vol. 25, Mark P. Zanna, ed. New York: Academic Press, 1-65. Schwartz, Shalom H. (2004), "Mapping and Interpreting Cultural Differences Around the World," in Comparing Cultures: Dimensions of Culture in a Comparative Perspective, H. Vinken, J. Soeters, and P. Ester, eds. Leiden, The Netherlands: Brill.

Schwartz, Shalom H., and Anat Bardi (2001), "Value Hierarchies Across Cultures: Taking a Similarities Perspective," Journal of Cross-Cultural Psychology, 32 (3), 268-90.

Shavitt, Sharon, Ashok K. Lalwani, Jing Zhang, and Carlos J. Torelli (2006), "The Horizontal/Vertical Distinction in Cross-Cultural 
Consumer Research,” Journal of Consumer Psychology, 16 (4), 325-56.

Shavitt, Sharon, Carlos J. Torelli, and Jimmy Wong (2009), "Identity-Based Motivation: Constraints and Opportunities in Consumer Research," Journal of Consumer Psychology, 19 (3), 261-66.

Skowronski, J.J., and D.E. Carlston (1989), "Negativity and Extremity Biases in Impression Formation: A Review of Explanations," Psychological Bulletin, 105 (1), 131-42.

Steenkamp, Jan-Benedict E.M., Rajeev Batra, and Dana L. Alden (2003), "How Perceived Brand Globalness Creates Brand Value," Journal of International Business Studies, 34 (1), 53-65.

Torelli, Carlos J., Alokparna Basu Monga, and Andrew M. Kaikati (2012), "Doing Poorly by Doing Good: Corporate Social Responsibility and Brand Concepts," Journal of Consumer Research, 38 (5), 948-63.

Torelli, Carlos J., and Andrew M. Kaikati (2009), "Values as Predictors of Judgments and Behaviors: The Role of Abstract and Concrete Mindsets," Journal of Personality and Social Psychology, 96 (1), 231-47.

Torelli, Carlos J., Ayşegül Özsomer, Sergio W. Carvalho, Hean Tat Keh, and Natalie Maehle (2012), "Brand Concepts as Representations of Human Values: Do Cultural Congruity and Compatibility Between Values Matter?' Journal of Marketing, 76 (4), 92-108.
Torelli, Carlos J., and Sharon Shavitt (2010), "Culture and Concepts of Power," Journal of Personality and Social Psychology, 99 (4), 703-23.

Van der Lans, Ralf, Yvonne van Everdingen, and Melnyk Valentyna (2016), "What to Stress, to Whom, and Where? A Cross-Country Investigation of the Effects of Perceived Brand Benefits on Buying Intentions," International Journal of Research in Marketing, 33 (4), 924-43.

Van de Vijver, Fons J., and Kwok Leung (1997), Methods and Data Analysis for Cross-Cultural Research. Thousand Oaks, CA: Sage Publications.

Wilkie, William (1994), Consumer Behavior, 3rd ed. New York: John Wiley \& Sons.

Yavas, Ugur, Bronislaw Verhage, and Robert T. Green (1992), "Global Consumer Segmentation Versus Local Market Orientation Empirical Findings," Management International Review, 32 (3), 263-72.

Zarantonello, Lia, Kamel Jedidi, and Bernd H. Schmitt (2013), "Functional and Experiential Routes to Persuasion: An Analysis of Advertising in Emerging Versus Developed Markets," International Journal of Research in Marketing, 30 (1), 46-56.

Zou, Shaoming, and S. Tamer Cavusgil (2002), "The GMS: A Broad Conceptualization of Global Marketing Strategy and Its Effect on Firm Performance," Journal of Marketing, 66 (4), 40-56. 
Copyright of Journal of Marketing Research (JMR) is the property of American Marketing Association and its content may not be copied or emailed to multiple sites or posted to a listserv without the copyright holder's express written permission. However, users may print, download, or email articles for individual use. 